\title{
Lag Synchronization in Coupled Multistable van der Pol-Duffing Oscillators
}

\author{
Dawid Dudkowski, Patrycja Kuzma, and Tomasz Kapitaniak \\ Division of Dynamics, Technical University of Lodz, Stefanowskiego 1/15, 90-924 Lodz, Poland \\ Correspondence should be addressed to Tomasz Kapitaniak; tomaszka@p.lodz.pl
}

Received 10 February 2014; Accepted 9 April 2014; Published 29 April 2014

Academic Editor: Jinde Cao

Copyright ( 2014 Dawid Dudkowski et al. This is an open access article distributed under the Creative Commons Attribution License, which permits unrestricted use, distribution, and reproduction in any medium, provided the original work is properly cited.

\begin{abstract}
We consider the system of externally excited identical van der Pol-Duffing oscillators unidirectionally coupled in a ring. When the coupling is introduced, each of the oscillator's trajectories is on different attractor. We study the changes in the dynamics due to the increase in the coupling coefficient. Studying the phase of the oscillators, we calculate the parameter value for which we obtain the antiphase lag synchronization of the system and also the bifurcation values for which we observe qualitative changes in the dynamics of already synchronized system. We give evidence that lag synchronization is typical for coupled multistable systems.
\end{abstract}

First studies about the synchronization of the mechanical systems date back to the 17 th century, when Christiaan Huygens watched two pendulum clocks hung on the common beam. As a result of the vibrations transmitted by this beam from one clock to the other, after a while they started to move with the same frequency. The huge development of science, especially the theory of the nonlinear dynamical systems, caused the expansion of the researches about different types of the synchronization phenomena. Today, the concept of the synchronization has applications in many areas of science.

In this paper we study the phenomenon known as the lag synchronization [1-15]. We have two cases of this phenomenon. In the in-phase lag synchronization, the state of the first coupled system at any moment of time $t$ is the same as the state of the second coupled system in moment $t-\tau$, where $\tau$ is a fixed lag. The case of the antiphase lag synchronization is more complicated. The dynamics is similar to the previously described one, but the values of the phase variables for the first system are opposite to the values of these variables for the second one. In our researches, we have observed the second type of the lag synchronization phenomenon. Lag synchronization has been observed mostly in chaotic [1-11] and hyperchaotic [12,13] coupled systems, but it has been even found in nonchaotic dynamics [15]. There are many papers about this phenomenon in Rössler systems $[1,9-12]$ and in various types of time delay systems [2-5, 13-15]. Papers [3-8] contain interesting results of this phenomenon in neuron systems. Works $[9,13]$ present how this type of synchronization turns into other types due to the changes in the system parameters. In our considerations, we focus not on the issue when and how the coupled system achieves the synchronization but on how it behaves after reaching it. We try to answer the question "how will the dynamics of the synchronized multistable oscillators change when the strength that couples them will be increased?"

As an example, we consider externally forced van der PolDuffing oscillator [16-33], which is multistable and has rare attractors [16]. The phenomenon of uncertainty due to the initial positions on the attractors has also been shown for this system [17]. In [18-22], considerations about various types of bifurcations for van der Pol-Duffing oscillator can be found. Most of the researches relate to chaotic behaviour of this system [23-26], but there are also considerations about periodic and quasiperiodic attractors [24]. The synchronization of coupled van der Pol-Duffing oscillators with various types of coupling and the time delay feedback have been described in [19, 25-33]. This multiplicity of properties ensures that each study on this system gives new, interesting results and extends the knowledge of nonlinear dynamics. 


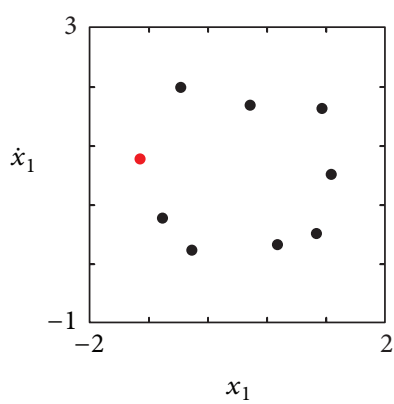

(a)

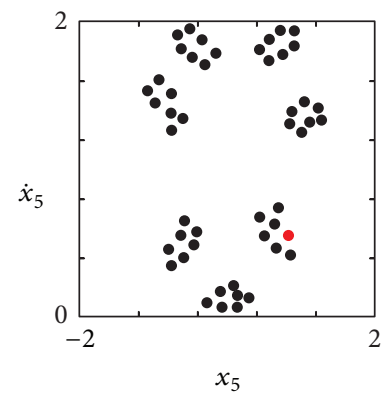

(e)

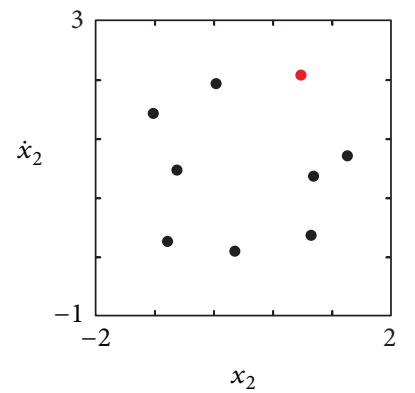

(b)

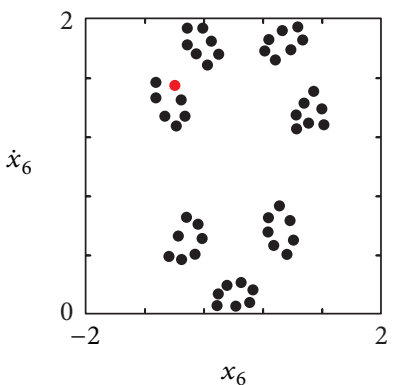

(f)

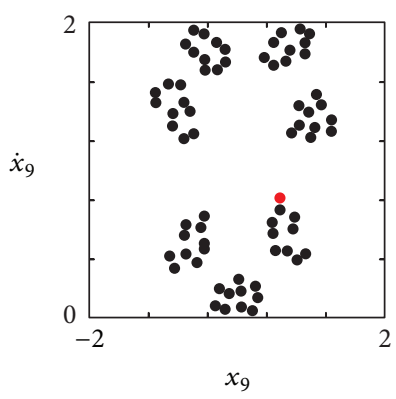

(i)

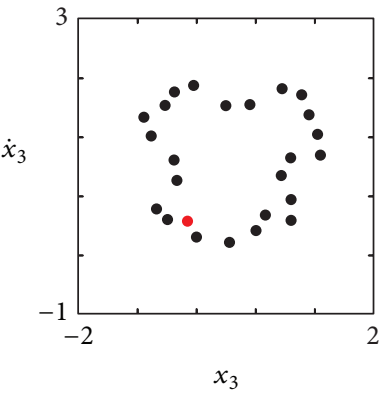

(c)

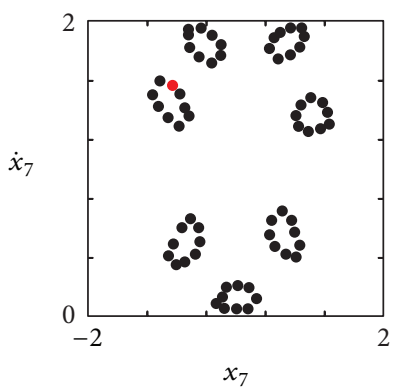

(g)

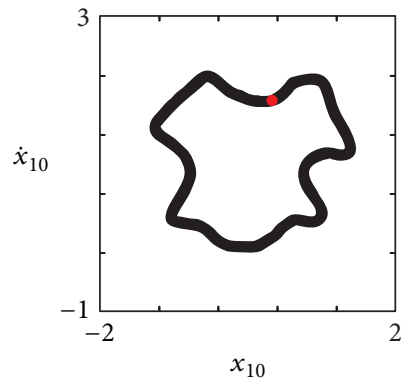

(j)

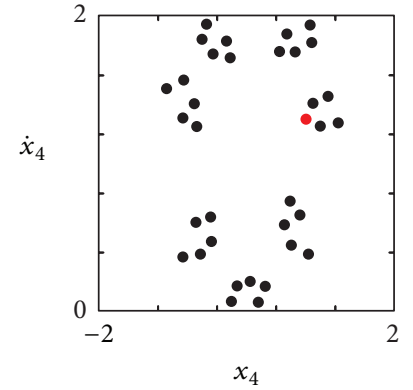

(d)

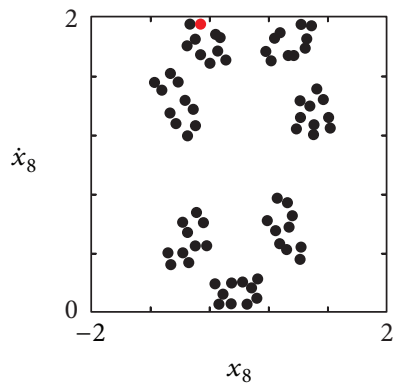

(h)

FIGURE 1: Poincaré maps of the subsystems of (1) for different initial conditions and $\varepsilon=0$. Maps (a)-(i) show periodic solutions, where the period is equal to number of dots on map multiplied by $2 \pi / 0.962$ (period of the excitation). The last map (j) represents quasiperiodic attractor.

In this paper, we consider ten externally excited identical van der Pol-Duffing oscillators unidirectionally coupled in a ring. The system is given by ten second-order ordinary differential equations:

$$
\begin{array}{r}
\ddot{x}_{1}-\alpha\left(1-x_{1}^{2}\right) \dot{x}_{1}+x_{1}^{3}=F \sin \omega t+\varepsilon\left(x_{1}-x_{10}\right), \\
\ddot{x}_{i}-\alpha\left(1-x_{i}^{2}\right) \dot{x}_{i}+x_{i}^{3}=F \sin \omega t+\varepsilon\left(x_{i}-x_{i-1}\right), \\
i=2, \ldots, 10,
\end{array}
$$

where $\alpha, \omega$, and $F$ are constant. The coupling between the 1st $\left(x_{1}\right)$ and the 10th $\left(x_{10}\right)$ oscillator implicates first equation. We assume that $\alpha=0.2, F=1$, and $\omega=0.962$ and consider $\varepsilon$ as a coupling coefficient.

In [16], it has been shown that single van der Pol-Duffing oscillator is multistable and that depending on the value of the parameter $\omega$ the attractors of different types coexist (i.e., periodic, quasiperiodic, and chaotic). In [16], the basins of attraction for some of these attractors are also contained. In our case, for $\omega=0.962$, we obtain periodic and quasiperiodic orbits.

At the beginning, when the coupling is introduced, the assumed initial conditions are presented as red dots on Poincaré maps in Figures 1(a)-1(j). For each map, points are taken from the trajectory of corresponding subsystem and the time interval between the consecutive values on the trajectory is equal to the period of the excitation $-2 \pi / 0.962$. Horizontal axes correspond to the $x_{i}$ coordinates, while vertical axes correspond to the $\dot{x}_{i}$ coordinates $(i=1, \ldots, 10)$. In this case, nine of the subsystems are on periodic trajectories and the 10 th one is on the quasiperiodic one.

We focus on determining the smallest value of the coupling coefficient $\varepsilon$ for which the oscillators of the system (1) synchronize. We will also study the qualitative changes in the dynamics of this system when we increase $\varepsilon$ parameter. To do this, the concept of the phase of the system is used. For the subsystem $i \in\{1, \ldots, 10\}$, we consider phase function $\varphi_{i}(t):=$ $\arctan \left(\dot{x}_{i}(t) / x_{i}(t)\right)$, where for $x_{i}(t)=0$ we assume $\varphi_{i}(t)=\pi / 2$ 


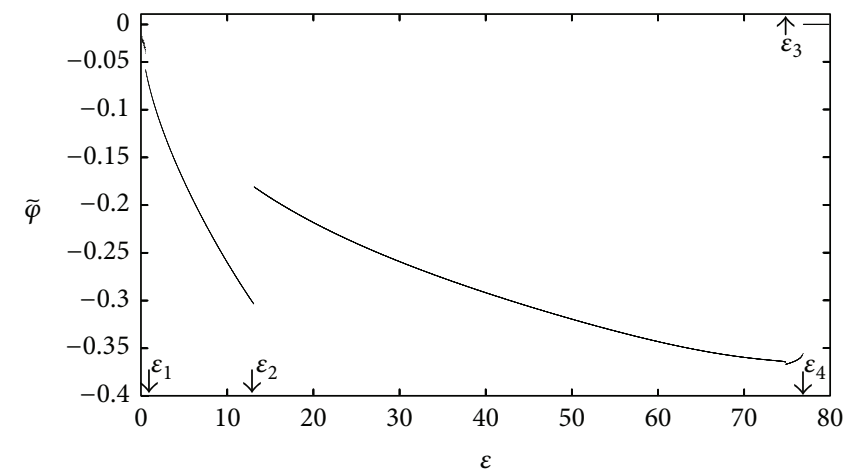

(a)
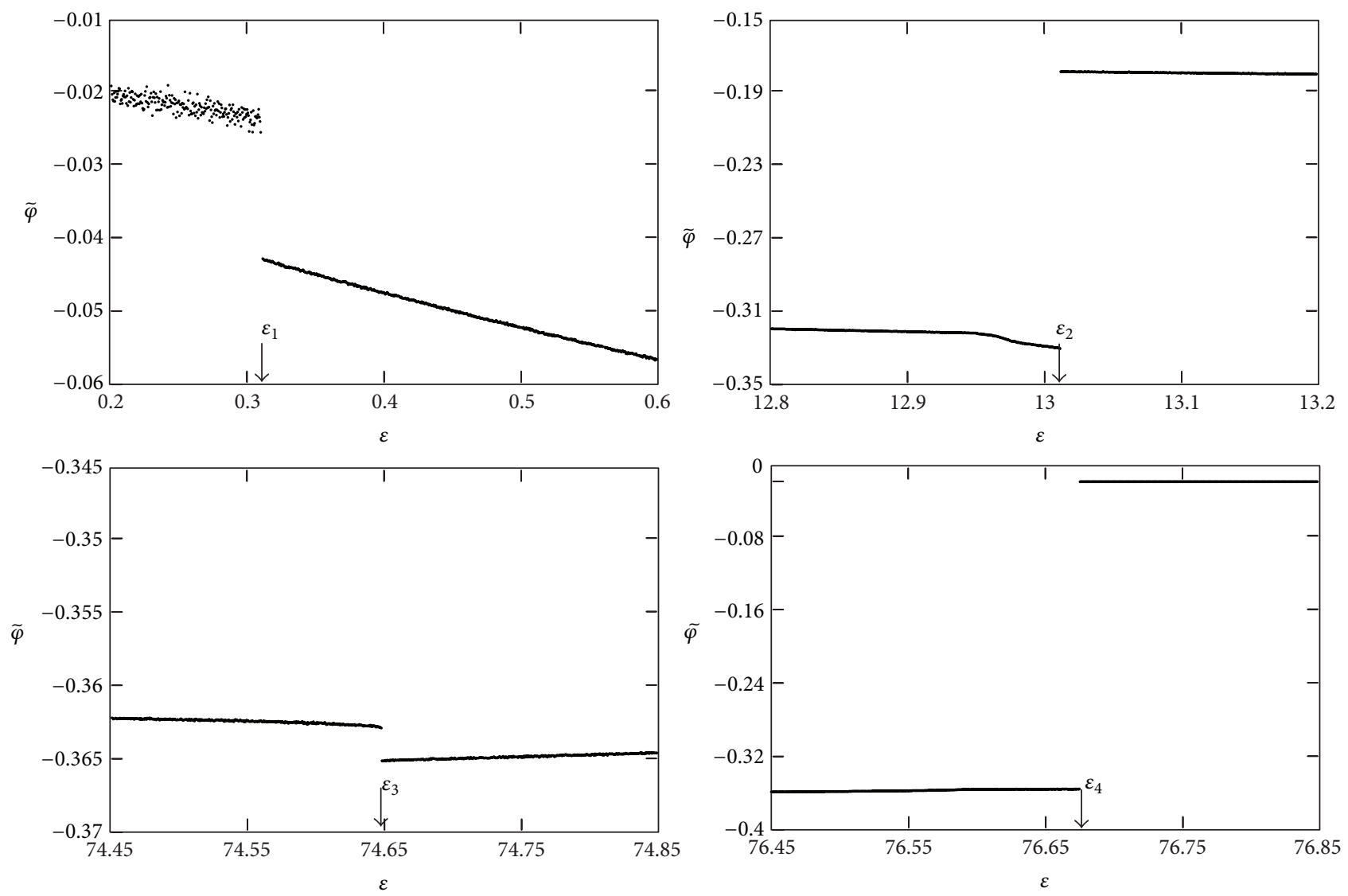

(b)

Figure 2: Mean phase $\widetilde{\varphi}$ of the 1st subsystem versus the coupling coefficient $\varepsilon$. On (b), changes of the $\widetilde{\varphi}$ in the small intervals around $\varepsilon_{1}, \varepsilon_{2}$, $\varepsilon_{3}$, and $\varepsilon_{4}$ are presented.

or $\varphi_{i}(t)=-\pi / 2$, depending on the sign of $\dot{x}_{i}(t)$. For special case $\dot{x}_{i}(t)=x_{i}(t)=0$, we assume $\varphi_{i}(t)=0$. For fixed $t$, if point $\left(x_{i}(t), \dot{x}_{i}(t)\right)$ is located in the 1 st or 4 th quadrant of twodimensional Cartesian system, then the above-mentioned function defines the angle on the plane between the half-line $[0, \infty)$ and the segment which begins in point $(0,0)$ and ends in point $\left(x_{i}(t), \dot{x}_{i}(t)\right)$. If point $\left(x_{i}(t), \dot{x}_{i}(t)\right)$ is located in the 2 nd or 3rd quadrant, then the function defines the angle between the half-line $(-\infty, 0]$ and the previously described segment $\overline{(0,0) ;\left(x_{i}(t), \dot{x}_{i}(t)\right)}$.
If two subsystems' trajectories are the same in phase space, or one of them is symmetrical across point $(0,0)$ to the second (i.e., the trajectories are on the same attractor or on attractors symmetrical across point $(0,0))$, then the mean values of the phases of these subsystems are equal. This fact is very helpful while examining the bifurcation diagrams of mean phase versus $\varepsilon$ for two oscillators. Overlapping of these diagrams suggests the synchronization of the subsystems. However, it should be emphasized that this fact is only a necessary but not sufficient condition for this phenomenon. 


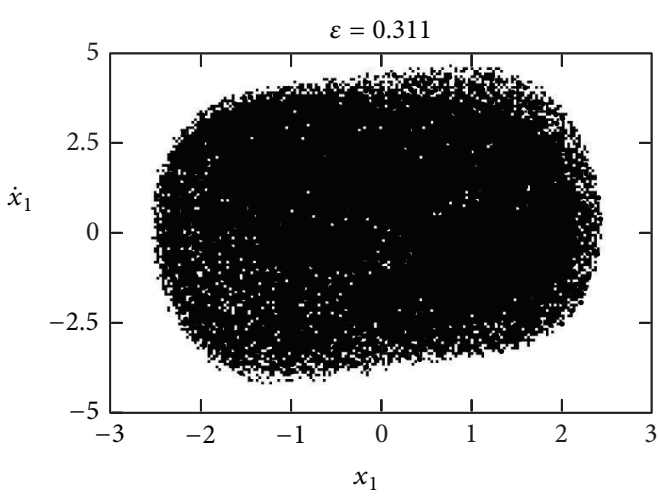

(a)

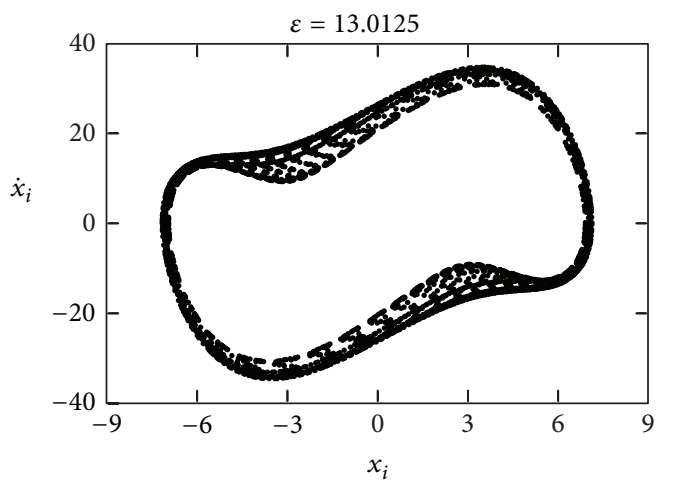

(c)

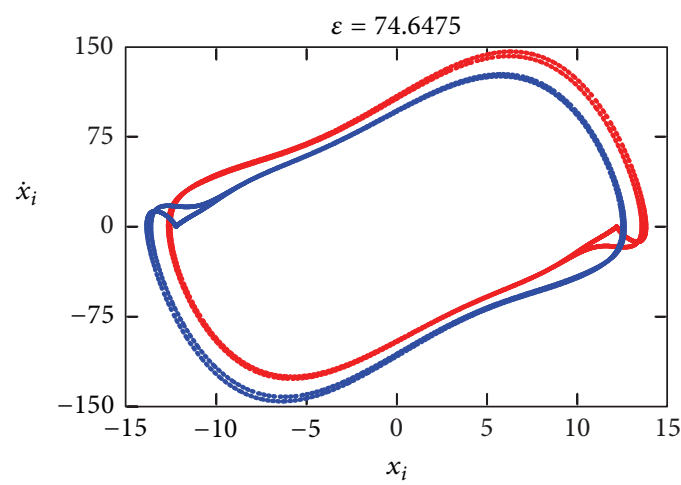

(e)

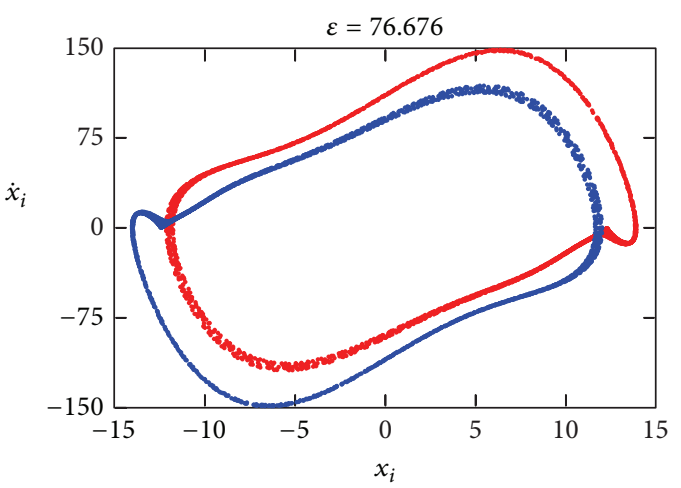

(g)

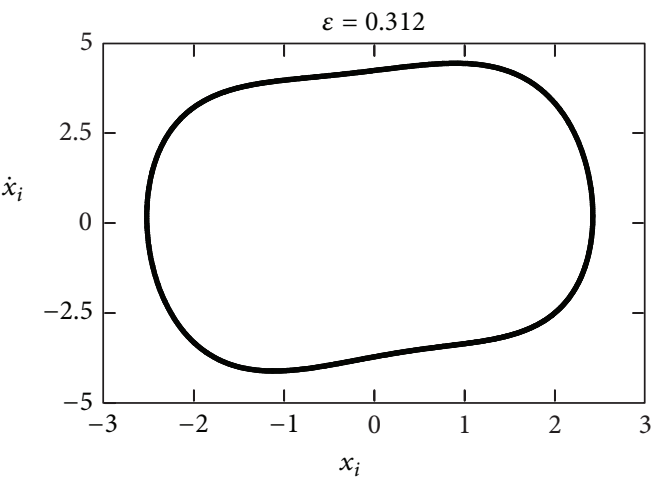

(b)

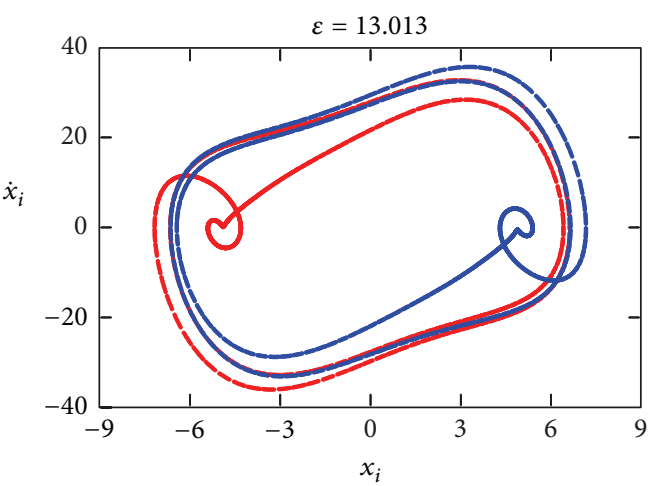

(d)

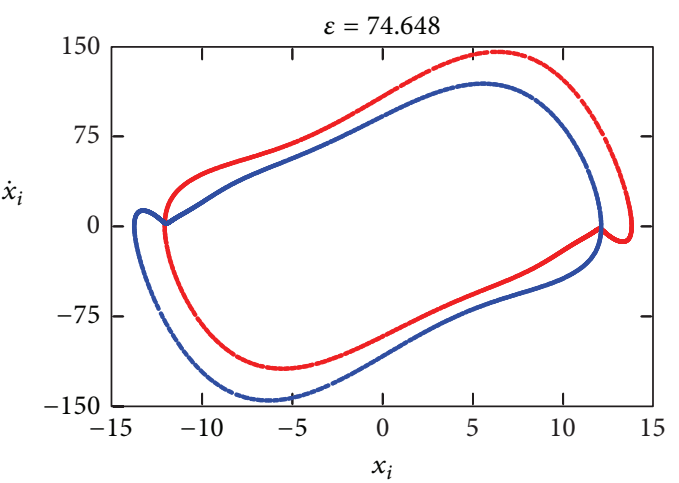

(f)

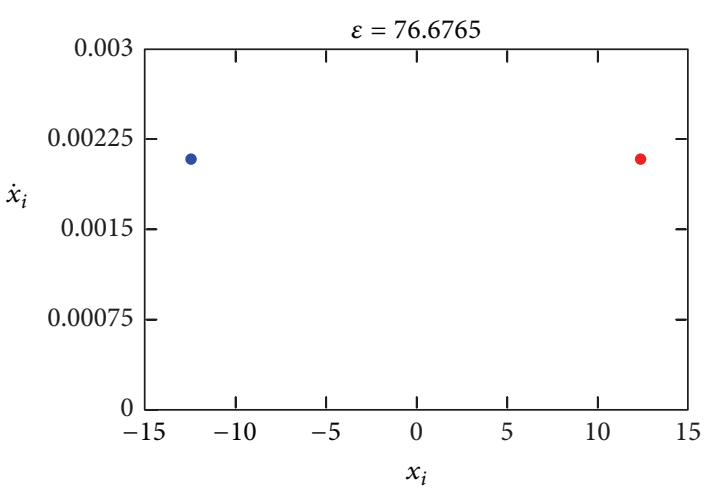

(h)

Figure 3: Poincaré maps of the subsystems of (1). On (a), the map for 1st oscillator is presented. Maps (b)-(c) refer to all of the subsystems (maps overlap). Maps (d)-(h) also refer to all of the oscillators, but maps of the 1st, 3rd, 5th, 7th, and 9th oscillators are marked by blue colour while maps for the $2 \mathrm{nd}$, 4 th, 6 th, 8 th, and 10 th by red colour. 
In Figure 2(a) the changes of the mean phase (denoted by $\widetilde{\varphi}$ ) of the 1st subsystem are presented. On horizontal axis are possible values of coupling coefficient in interval $[0,80]$ and on vertical axis the values of $\tilde{\varphi}$. The bifurcation points are marked as $\varepsilon_{1}, \varepsilon_{2}, \varepsilon_{3}$, and $\varepsilon_{4}$. In Figure $2(\mathrm{~b})$ the changes in the small intervals around this bifurcation are shown.

The changes in the dynamics of the subsystems in bifurcation points are shown on Poincaré maps in Figure 3. The actual value of $\varepsilon$ parameter for which we calculate the map is above each of them.

When $\varepsilon \in\left[0, \varepsilon_{1}\right)$ the $\widetilde{\varphi}$ fluctuates, but after reaching $\varepsilon_{1}$ it changes continuously (except bifurcations in $\varepsilon_{2}, \varepsilon_{3}$, and $\varepsilon_{4}$ ). This behaviour is common for all of the oscillators; that is, the diagrams calculated for all of the subsystems overlap for $\varepsilon \in\left(\varepsilon_{1}, 80\right]$. For coupling coefficient equal to $\varepsilon_{1}$, we obtain the synchronization of the system. Indeed, Poincare map for $\varepsilon=0.311$ represents chaotic behaviour of the oscillators (Figure 3 shown for the 1st one) but then for $\varepsilon=0.312$ all of them are synchronized on one common quasiperiodic attractor. The described behaviour continues for $\varepsilon \in\left[\varepsilon_{1}, \varepsilon_{2}\right)$. It changes for $\varepsilon_{2}$ when the common attractor is destroyed and two symmetrical attractors appear. For $\varepsilon=13.0125$ subsystems are on one attractor and for $\varepsilon=13.013$ they group equally on two tori. This continues for $\varepsilon \in\left[\varepsilon_{2}, \varepsilon_{3}\right)$ and for $\varepsilon_{3}$ we obtain another bifurcation known as the doubling of torus $[34,35]$. Previously, subsystems are grouped equally on the attractors and for $\varepsilon \in\left[\varepsilon_{3}, \varepsilon_{4}\right)$ there are no qualitative changes in the dynamics. Finally, in $\varepsilon_{4}$ we obtain the critical moment when the coupling coefficient is so large that the dynamics is caused only by the external force and so the oscillators vibrate periodically (with the period of the excitation). In Figure 3, for $\varepsilon=76.676$ two quasiperiodic attractors coexist and for $\varepsilon=76.6765$ we obtain a single point on Poincaré map for each subsystem. From now on, no matter how much $\varepsilon$ will be increased, the dynamics of the system does not change.

It is also worth mentioning that when the oscillators are synchronized for the fixed value of $\varepsilon$, the lag in the synchronization between them can be easily numerically found by analyzing the differences in positions of coupled subsystems. Here, we considered the function $\delta(\tau):=\max \left\{\mid x_{i}(t)-\right.$ $\left.\left(-x_{i+1}(t+\tau)\right) \mid: t \geq t_{s}\right\}$, where $i \in\{1, \ldots, 10\}$ is the number of one of the oscillators and $t_{s}$ is the time moment from which the subsystems are synchronized. For fixed argument, this function describes the value of maximum difference between positions of corresponding oscillators in the whole time interval. The position of the first oscillator is taken in time $t$ while the position of the second one in time $t+\tau$. The lag value $\tau$ in the synchronization is the argument for which the function value $\delta(\tau)$ is sufficiently small. We made calculations for $\tau \in[0,100]$ and obtained the following results: for $\varepsilon=0.312$ the lag is $\tau=22.84$ and $\delta(\tau)=0.0102$; for $\varepsilon=13.013$ the lag is $\tau=42.485$ and $\delta(\tau)=0.022$; for $\varepsilon=$ 74.648 the lag is $\tau=87.36$ and $\delta(\tau)=0.0215$; for $\varepsilon=76.6765$ the lag is $\tau=3.265$ and $\delta(\tau)=0$ (ideal lag synchronization).

To summarize, by coupling the van der Pol-Duffing oscillators and properly selecting the value of the coupling coefficient we observe the lag synchronization of the oscillators. As mentioned in the preliminaries, the dynamics of the single system is very rich and this is the main reason why simple complete synchronization is not obtained. We have determined the critical value for which the phenomenon occurs $\left(\varepsilon_{1}\right)$ and examined the changes in the dynamics while increasing the coupling coefficient. The common attractor on which all subsystems synchronize due to increase in parameter $\varepsilon$ changes not only quantitatively but also qualitatively. Single torus, common to all of the oscillators, due to bifurcations occurring for $\varepsilon_{2}, \varepsilon_{3}$, and $\varepsilon_{4}$ converts into two double tori, then into two single tori, and finally into periodic orbits. The observed phenomena are robust as they can be observed for the wide range of system parameters.

The obtained results suggest that when coupling the systems that have similar properties as van der Pol-Duffing oscillator, the characteristics of the structure that couples the subsystems should be selected very carefully (e.g., it may be a spring and its coefficient of elasticity). Apart from basic cases when this coefficient reaches the smallest value for which the system synchronizes and a large enough value for which the subsystems begin to oscillate at a frequency of an external force, there is a range of intermediate values of this coefficient that cause the qualitative changes in the dynamics of the synchronized system. This fact should be taken into account while constructing certain mechanical models. We argue that the lag synchronization is typical for coupled multistable systems.

\section{Conflict of Interests}

The authors declare that there is no conflict of interests regarding the publication of this paper.

\section{Acknowledgments}

This work has been supported by the Foundation for Polish Science, Team Programme, Project no. TEAM/2010/5/5, and by the Polish National Science Centre, MAESTRO Programme, Project no. 2013/08/A/ST8/00/780.

\section{References}

[1] D. Pazo, M. A. Zaks, and J. Kurths, "Role of unstable periodic orbits in phase and lag synchronization between coupled chaotic oscillators," Chaos. An Interdisciplinary Journal of Nonlinear Science, vol. 13, no. 1, pp. 309-318, 2003.

[2] C. Li, X. Liao, and R. Zhang, "A unified approach for impulsive lag synchronization of chaotic systems with time delay," Chaos, Solitons \& Fractals, vol. 23, no. 4, pp. 1177-1184, 2005.

[3] C. Li, X. Liao, and K.-w. Wong, "Chaotic lag synchronization of coupled time-delayed systems and its applications in secure communication," Physica D. Nonlinear Phenomena, vol. 194, no. 3-4, pp. 187-202, 2004.

[4] Y. Sun and J. Cao, "Adaptive lag synchronization of unknown chaotic delayed neural networks with noise perturbation," Physics Letters A: General, Atomic and Solid State Physics, vol. 364, no. 3-4, pp. 277-285, 2007.

[5] Y. Yang and J. Cao, "Exponential lag synchronization of a class of chaotic delayed neural networks with impulsive effects," Physica 
A: Statistical Mechanics and its Applications, vol. 386, no. 1, pp. 492-502, 2007.

[6] J. Cao, A. Alofi, A. Al-Mazrooei, and A. Elaiw, "Synchronization of switched interval networks and applications to chaotic neural networks," Abstract and Applied Analysis, vol. 2013, Article ID 940573, 11 pages, 2013.

[7] X. Yang, J. Cao, Y. Long, and W. Rui, "Adaptive lag synchronization for competitive neural networks with mixed delays and uncertain hybrid perturbations," IEEE Transactions on Neural Networks, vol. 21, no. 10, pp. 1656-1667, 2010.

[8] J. Cao and Y. Wan, "Matrix measure strategies for stability and synchronization of inertial BAM neural network with time delays," Neural Networks, vol. 53, pp. 165-172, 2014.

[9] M. G. Rosenblum, A. S. Pikovsky, and J. Kurths, "From phase to lag synchronization in coupled chaotic oscillators," Physical Review Letters, vol. 78, no. 22, pp. 4193-4196, 1997.

[10] S. Boccaletti and D. L. Valladares, "Characterization of intermittent lag synchronization," Physical Review E-Statistical Physics, Plasmas, Fluids, and Related Interdisciplinary Topics, vol. 62, no. 5, pp. 7497-7500, 2000.

[11] S. Taherion and Y. Lai, "Observability of lag synchronization of coupled chaotic oscillators," Physical Review E-Statistical Physics, Plasmas, Fluids, and Related Interdisciplinary Topics, vol. 59, no. 6, pp. R6247-R6250, 1999.

[12] C. Li, X. Liao, and K.-w. Wong, "Lag synchronization of hyperchaos with application to secure communications," Chaos, Solitons \& Fractals, vol. 23, no. 1, pp. 183-193, 2005.

[13] D. V. Senthilkumar and M. Lakshmanan, "Transition from anticipatory to lag synchronization via complete synchronization in time-delay systems," Physical Review E-Statistical, Nonlinear, and Soft Matter Physics, vol. 71, no. 1, Article ID 016211, 2005.

[14] W. Yu and J. Cao, "Adaptive synchronization and lag synchronization of uncertain dynamical system with time delay based on parameter identification," Physica A: Statistical Mechanics and its Applications, vol. 375, no. 2, pp. 467-482, 2007.

[15] E. M. Shahverdiev, S. Sivaprakasam, and K. A. Shore, "Lag synchronization in time-delayed systems," Physics Letters A: General, Atomic and Solid State Physics, vol. 292, no. 6, pp. 320324, 2002.

[16] A. Chudzik, P. Perlikowski, A. Stefanski, and T. Kapitaniak, "Multistability and rare attractors in van der Pol-Duffing oscillator," International Journal of Bifurcation and Chaos in Applied Sciences and Engineering, vol. 21, no. 7, pp. 1907-1912, 2011.

[17] P. Kuzma, M. Kapitaniak, and T. Kapitaniak, "Coupling multistable systems: uncertainty due to the initial positions on the attractors," Journal of Theoretical and Applied Mechanics, vol. 52, no. 1, pp. 281-284, 2014.

[18] W. Yu and J. Cao, "Hopf bifurcation and stability of periodic solutions for van der Pol equation with time delay," Nonlinear Analysis. Theory, Methods \& Applications A: Theory and Methods, vol. 62, no. 1, pp. 141-165, 2005.

[19] S. Ma, Q. Lu, and Z. Feng, "Double Hopf bifurcation for van der Pol-Duffing oscillator with parametric delay feedback control," Journal of Mathematical Analysis and Applications, vol. 338, no. 2, pp. 993-1007, 2008.

[20] J. C. Ji, "Nonresonant Hopf bifurcations of a controlled van der Pol-Duffing oscillator," Journal of Sound and Vibration, vol. 297, no. 1-2, pp. 183-199, 2006.

[21] A. Algaba, E. Freire, E. Gamero, and A. J. Rodríguez-Luis, "Analysis of Hopf and Takens-Bogdanov bifurcations in a modified van der Pol-Duffing oscillator," Nonlinear Dynamics, vol. 16, no. 4, pp. 369-404, 1998.

[22] A. Algaba, E. Freire, E. Gamero, and A. J. Rodríguez-Luis, "A tame degenerate Hopf-pitchfork bifurcation in a modified van der Pol-Duffing oscillator," Nonlinear Dynamics, vol. 22, no. 3, pp. 249-269, 2000.

[23] F. M. Moukam Kakmeni, S. Bowong, C. Tchawoua, and E. Kaptouom, "Strange attractors and chaos control in a Duffingvan der Pol oscillator with two external periodic forces," Journal of Sound and Vibration, vol. 277, no. 4-5, pp. 783-799, 2004.

[24] W. Szemplińska-Stupnicka and J. Rudowski, “The coexistence of periodic, almost-periodic and chaotic attractors in the van der Pol-Duffing oscillator," Journal of Sound and Vibration, vol. 199, no. 2, pp. 165-175, 1997.

[25] H. Fotsin, S. Bowong, and J. Daafouz, "Adaptive synchronization of two chaotic systems consisting of modified Van der PolDuffing and Chua oscillators," Chaos, Solitons and Fractals, vol. 26, no. 1, pp. 215-229, 2005.

[26] R. Yamapi and G. Filatrella, "Strange attractors and synchronization dynamics of coupled Van der Pol-Duffing oscillators," Communications in Nonlinear Science and Numerical Simulation, vol. 13, no. 6, pp. 1121-1130, 2008.

[27] A. P. Kuznetsov, N. V. Stankevich, and L. V. Turukina, "Coupled van der Pol-Duffing oscillators: phase dynamics and structure of synchronization tongues," Physica D. Nonlinear Phenomena, vol. 238, no. 14, pp. 1203-1215, 2009.

[28] A. P. Kuznetsov and J. P. Roman, "Properties of synchronization in the systems of non-identical coupled van der Pol and van der Pol-Duffing oscillators. Broadband synchronization," Physica D. Nonlinear Phenomena, vol. 238, no. 16, pp. 1499-1506, 2009.

[29] H. G. Enjieu Kadji and R. Yamapi, "General synchronization dynamics of coupled Van der Pol-Duffing oscillators," Physica A: Statistical Mechanics and its Applications, vol. 370, no. 2, pp. 316-328, 2006.

[30] X. Li, J. C. Ji, C. H. Hansen, and C. Tan, "The response of a Duffing-van der Pol oscillator under delayed feedback control," Journal of Sound and Vibration, vol. 291, no. 3-5, pp. 644-655, 2006.

[31] Z. Wang, Z. Duan, and J. Cao, "Impulsive synchronization of coupled dynamical networks with nonidentical Duffing oscillators and coupling delays," Chaos, vol. 22, no. 1, Article ID 013140, 2012.

[32] A. Maccari, "Vibration amplitude control for a van der PolDuffing oscillator with time delay," Journal of Sound and Vibration, vol. 317, no. 1-2, pp. 20-29, 2008.

[33] J. Xu and K. W. Chung, "Effects of time delayed position feedback on a van der Pol-Duffing oscillator," Physica D. Nonlinear Phenomena, vol. 180, no. 1-2, pp. 17-39, 2003.

[34] S.-Y. Kim, W. Lim, and Y. Kim, "Torus doublings in symmetrically coupled period-doubling systems," Journal of the Korean Physical Society, vol. 56, no. 31, pp. 963-968, 2010.

[35] K. Kaneko, "Doubling of torus," Progress of Theoretical Physics, vol. 69 , no. 6, pp. 1806-1810, 1983. 


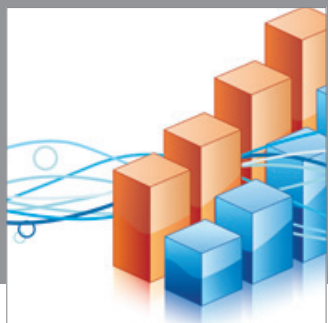

Advances in

Operations Research

mansans

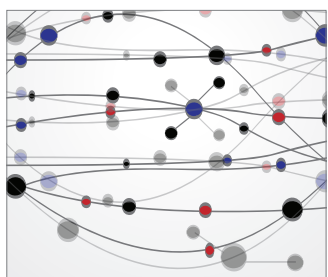

The Scientific World Journal
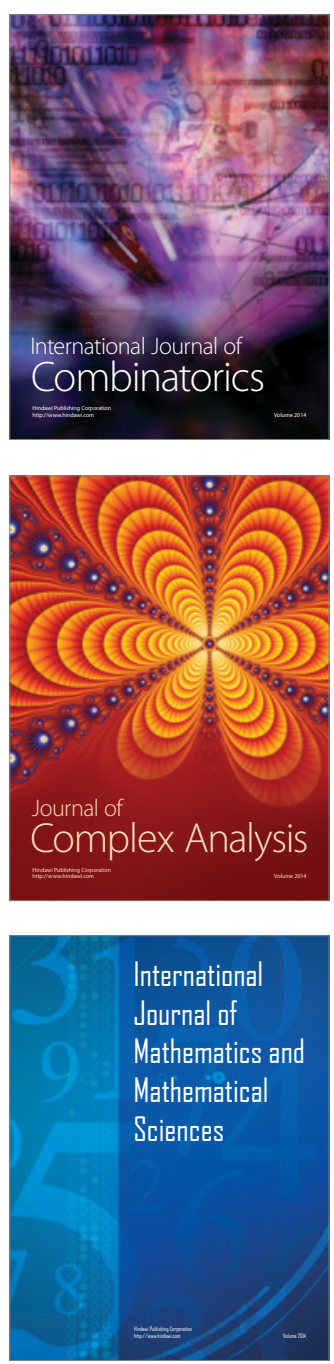
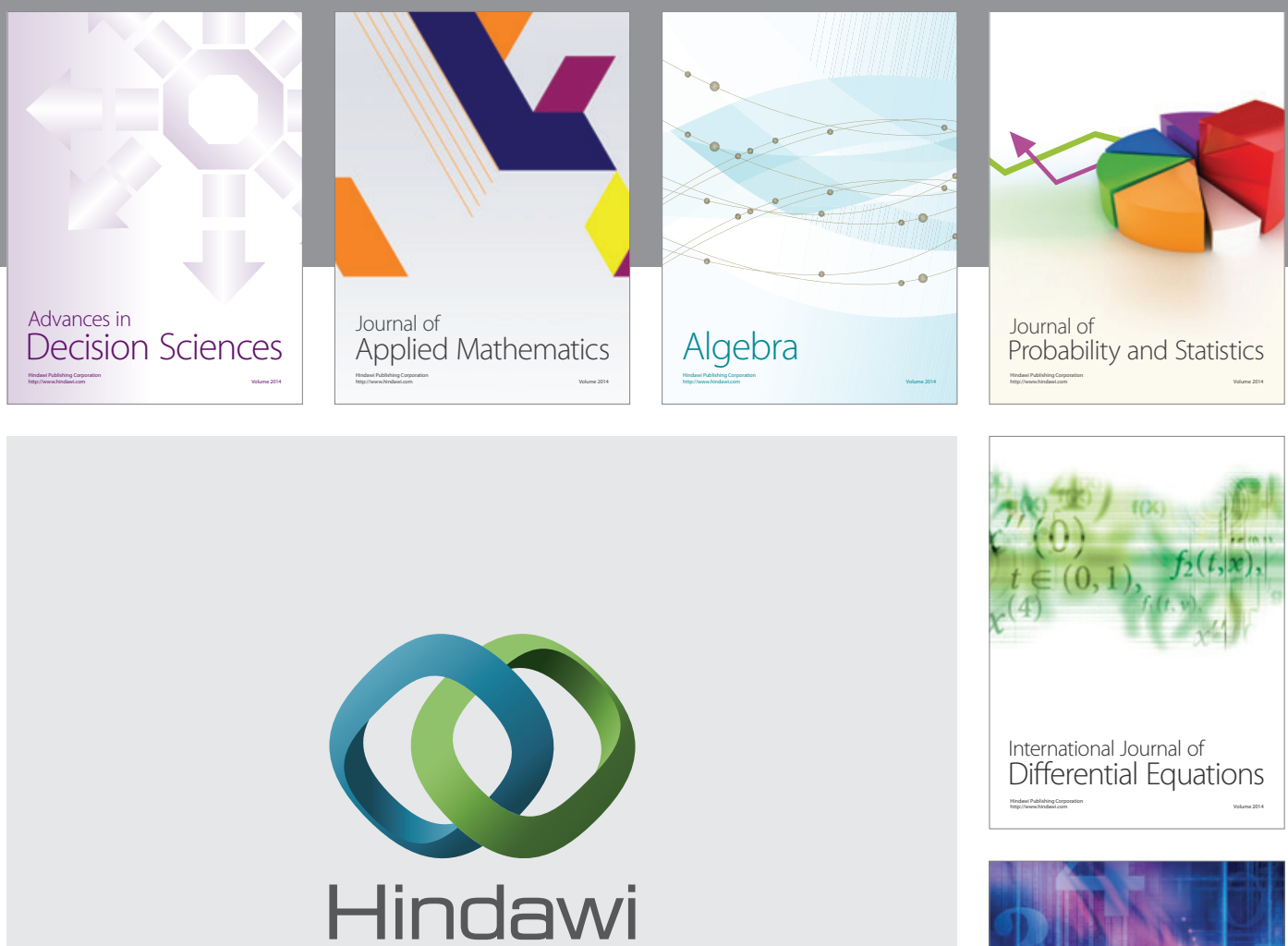

Submit your manuscripts at http://www.hindawi.com
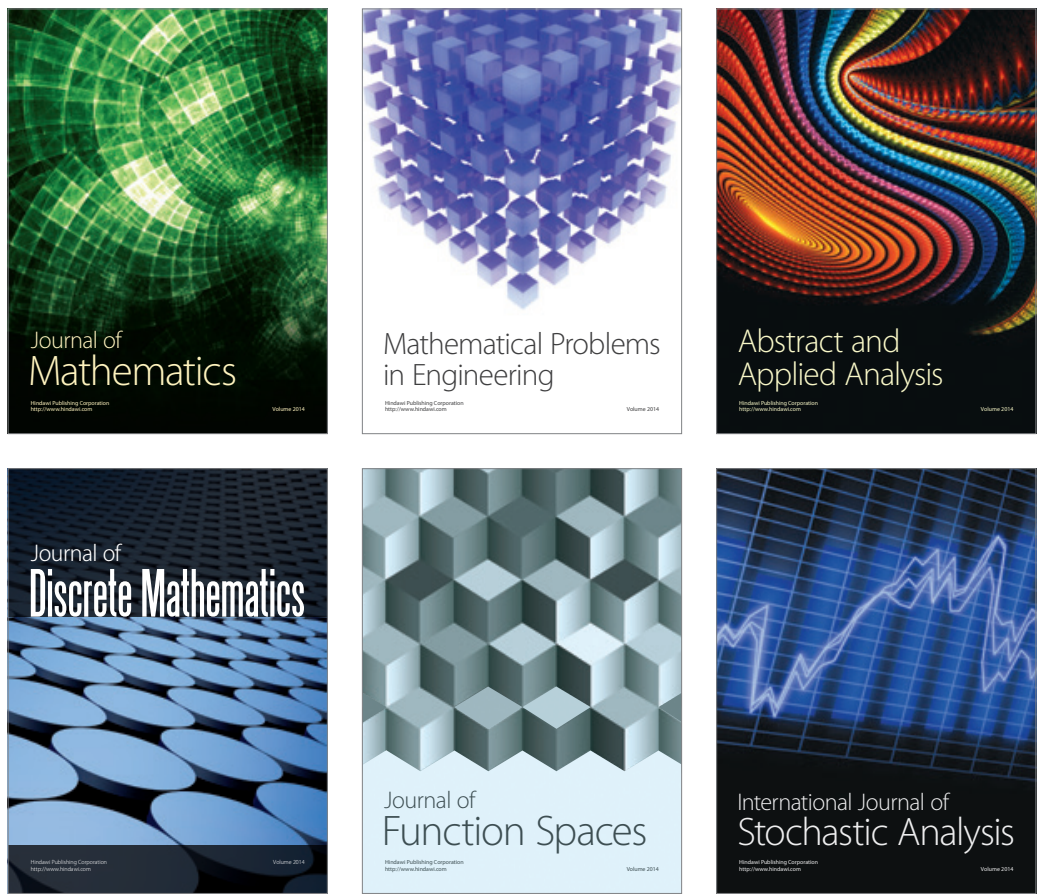

Journal of

Function Spaces

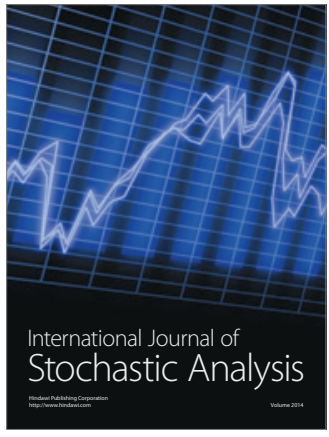

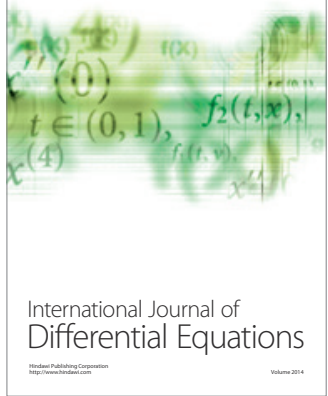
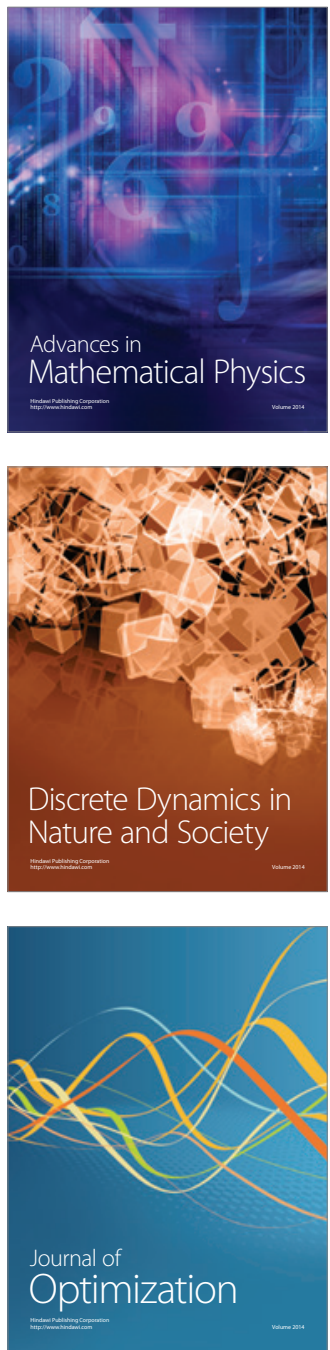